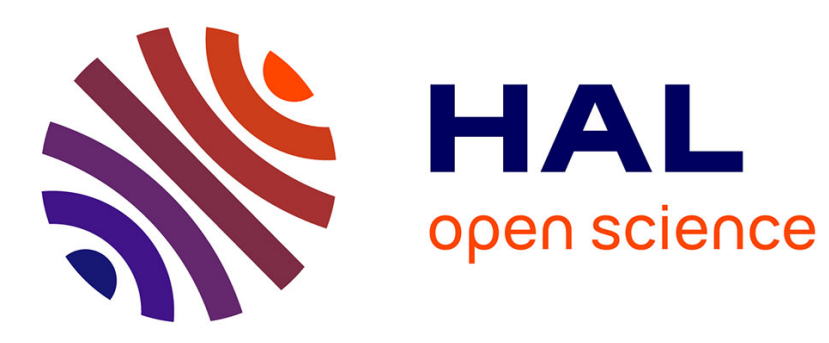

\title{
Nerve Detection in Ultrasound Images Using Median Gabor Binary Pattern
}

\author{
Oussama Hadjerci, Adel Hafiane, Pascal Makris, Donatello Conte, Pierre \\ Vieyres, Alain Delbos
}

\section{- To cite this version:}

Oussama Hadjerci, Adel Hafiane, Pascal Makris, Donatello Conte, Pierre Vieyres, et al.. Nerve Detection in Ultrasound Images Using Median Gabor Binary Pattern. 11th International Conference, ICIAR 2014, Oct 2014, Villamoura, Portugal. pp.132 - 140, 10.1007/978-3-319-11755-3_15 . hal-01083075

\section{HAL Id: hal-01083075 \\ https://hal.science/hal-01083075}

Submitted on 15 Nov 2014

HAL is a multi-disciplinary open access archive for the deposit and dissemination of scientific research documents, whether they are published or not. The documents may come from teaching and research institutions in France or abroad, or from public or private research centers.
L'archive ouverte pluridisciplinaire HAL, est destinée au dépôt et à la diffusion de documents scientifiques de niveau recherche, publiés ou non, émanant des établissements d'enseignement et de recherche français ou étrangers, des laboratoires publics ou privés. 


\title{
Nerve detection in ultrasound images using median Gabor binary pattern
}

\author{
Oussama Hadjerci ${ }^{1}$, Adel Hafiane ${ }^{1}$, Pascal Makris ${ }^{2}$, Donatello Conte ${ }^{2}$, Pierre \\ Vieyres $^{3}$, and Alain Delbos ${ }^{4}$ \\ 1 INSA Centre Val de Loire, University of Orléans, Laboratory PRISME EA 4229, \\ 88 boulevard Lahitolle, F-18020 Bourges, France. \\ 2 University of Francois Rabelais, Laboratory LI EA 6300, \\ 64 avenue Jean Portalis, F-37200 Tours, France. \\ 3 University of Orléans, Laboratory PRISME EA 4229, \\ 63 Avenue de Lattre de Tassigny F-18020 Bourges, France. \\ 4 Clinique Medipole Garonne, \\ 45 rue de Gironis CS 13624, F-31036 Toulouse, France.
}

\begin{abstract}
Ultrasound in regional anesthesia (RA) has increased in popularity over the last years. The nerve localization presents a key step for RA practice, it is therefore valuable to develop a tool able to facilitate this practice. The nerve detection in the ultrasound images is a challenging task, since the noise and other artifacts corrupt the visual properties of such kind of tissue. In this paper we propose a new method to address this problem. The proposed technique operates in two steps. As the median nerve belongs to a hyperechoic region, the first step consists in the segmentation of this type of region using the k-means algorithm. The second step is more critical; it deals with nerve structure detection in noisy data. For that purpose, a new descriptor is developed. It combines tow methods median binary pattern (MBP) and Gabor filter to obtain the median Gabor binary pattern (MGBP). The method was tested on 173 ultrasound images of the median nerve obtained from three patients. The results showed that the proposed approach achieves better accuracy than the original MBP, Gabor descriptor and other popular descriptors.
\end{abstract}

Keywords: image segmentation, feature extraction, image texture analysis, supervised learning, nerve detection, regional anesthesia.

\section{Introduction}

Regional anesthesia (RA) is performed by injecting the anesthetic close to a nerve block to immobilize a part of human body. Ultrasound imaging is an essential way in the practice of RA, it allows the visualization of the anatomical tissues, and it facilitates the needle control [19]. However, RA is a complex technique that requires a long learning process and years of experience $[19,10]$. Hence, it is important to provide anesthetists with a tool based on ultrasound images to improve the RA practice. 
Segmentation and detection in ultrasound (US) images have been applied to several applications such as abdomen, breast, liver, kidney, etc $[14,17,7,4]$. Very few works have studied the automatic nerve detection in the US images [16]. The nerve region is not a salient structure in the ultrasound images, due to the poor quality of the US imaging modality that generates many effects such as speckle noise, signal degradation, artifacts, etc [11]. Indeed, the segmentation and recognition in the US images are among the most challenging problems in the field of image processing and analysis. Numerous segmentation approaches, such as classification, active contour and graph cuts, have been used to tackle this issue [11]. According to the type of application, these methods require choosing relevant features to detect the regions of interest. Texture information is an important cue for many applications including the nerve blocks detection. However, texture analysis in US images is not an easy task. Nevertheless, several works have shown useful properties using texture to segment different tissues in US images. Features based on gray level correlation matrix (GLCM) [6], have been used for the detection of cardiac images [1], and the placenta [9]. In another work, GLCM has been utilized to guide the evolution of active contour for detecting the Thyroid Gland [15]. Recently the wavelet approach has been used with support vector machine (SVM) classifier to detect the prostate region [20], and also thresholding based wavelet has been used to remove the noise to detect the prostate [8]. In [13] Gabor filter bank has been combined with active contours for the prostate detection. Gabor filter is also utilized with expectation and maximization algorithm to detect liver and cystic kidney [7]. The local binary pattern (LBP) has been also utilized in the purpose of the prostate segmentation [4]. The performance of texture-based methods depends on the type of the tissue in the US images. Most of the state-of-the-art techniques have been applied to either hyperechoic or hypoechoic regions. The textural aspect of these regions is different each other, which requires different approaches. The nerve region presents a particular pattern that can be hypoechoic or hyperechoic structure, depending on the size of the nerve, the probe frequency and the angle of the ultrasound beam [10]. In this work, we address the problem of "median nerve" detection that presents a hyperechoic structure with a particular textural information. Due to the poor quality of the US images the nerve region is not easy to distinguish among others anatomical structures. The traditional texture features are not be sufficient to handle such a situation. In this paper, we propose a new method for detecting the median nerve. This method consists in two phases. The first one separates the foreground regions (hyperechoic tissues). The second phase extracts the nerve area in the foreground using support vector machine (SVM) and a new type of features that is a combination between Gabor filter and median binary patterns (MBP) [5]. Fig. 1 shows the flowchart of the proposed technique. 


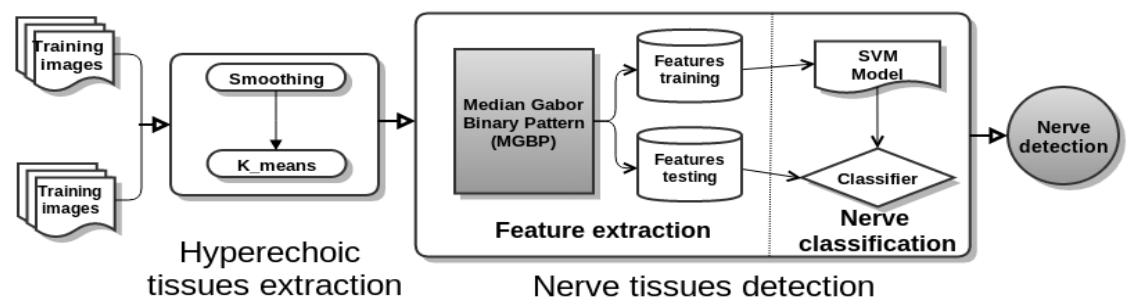

Fig. 1. Flowchart of the nerve detection in the ultrasound images.

\section{Nerve Detection}

The median nerve appears brighter in the US images since it is a hyperechoic structure. Therefore, the first step requires the segmentation of the hyperechoic foreground tissues to reduce the non useful information. In the next stage, the nerve is extracted from the foreground using texture features, called Median Gabor Binary Pattern (MGBP).

\subsection{Hyperechoic tissues segmentation}

To extract the hyperechoic tissues, we used Gaussian filtering processing to reduce the image noise, then k-means algorithm is applied to classify each pixel into background and foreground regions. Fig. 2 shows an example of the segmentation result, where we can distinguish the hyperechoic tissues from the other ones.

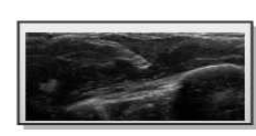

Input image

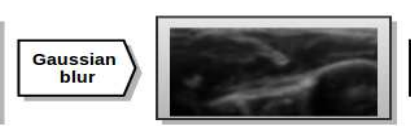

Gaussian filter response

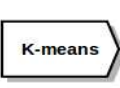

K-means

Clustering result

Fig. 2. Segmentation of hyperhecoic tissues using K-means.

\section{$2.2 \quad$ Feature extraction}

As we are dealing with noisy data, it is important for the descriptor not to be sensitive to the noise. The Gabor descriptor can help to reduce the noise effect, since it is based on band-pass filtering process [3]. However, frequency methods such as Gabor filter are not sufficient to characterize the nerve structure, it is more interesting to use additional information. The LBP based methods are among the best techniques for texture analysis and it can be combined with Gabor descriptors to achieve better performances as shown in [21]. This method called 
Local Gabor Binary Pattern (LGBP) has demonstrated some robustness with respect to the noise, while producing good performances for recognition task. LGBP can be suitable for ultrasound images, however it may fail to characterize the nerve texture due to the importance of the noise. Median Binary Pattern (MPB) has more interesting properties when it deals with noisy textures. MBP incorporates the median filtering processing which helps to reduce the noise. We propose to combine MBP with Gabor Filters producing the Median Gabor Binary Pattern (MGBP) descriptor, as shown in Fig. 3.

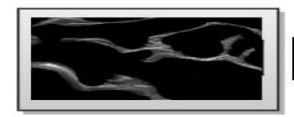

Input image
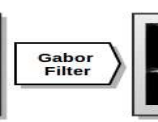

Gabor response

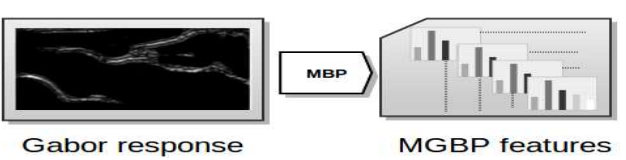

Fig. 3. The stage of MGBP feature extraction method.

To compute the $M G B P$ at the position $(x, y)$ on a given image $I$, first, $I$ is convolved with Gabor kernel, expressed by the following equation,

$$
g(x, y)=\exp \left[-\frac{1}{2}\left(\frac{x^{\prime 2}}{\sigma_{x}^{2}}+\frac{y^{\prime 2}}{\sigma_{y}^{2}}\right)\right] \cos \left(2 \pi f x^{\prime}\right)
$$

where $x^{\prime}=x \cos \phi+y \sin \phi, y^{\prime}=-x \sin \phi+y \cos \phi, \phi$ is the filter direction, $\sigma$ is the standard deviation of Gaussian envelope, and $f$ is the frequency of cosine wave. We use the magnitude of the Gabor filter response, that is $z(x, y)=$ $|I(x, y) * g(x, y)|$. The MGBP is determined by mapping from the response $z(x, y)$ space to a localized binary pattern, thereby thresholding the neighborhood pixels against their median value. The MGBP at pixel $(x, y)$ is defined as,

$$
M G B P_{P, R}=\sum_{p=0}^{P-1} \delta\left(z_{p}-z_{m}\right) 2^{P} \delta=\left\{\begin{array}{l}
1, x \geqslant 0 \\
0, x<0
\end{array}\right.
$$

We adopted the same strategy as LBP and MBP [12,5] for the different parameters, where $P$ is the number of neighbors and $R$ is the radius of the circular neighborhood, $z_{p}$ is the pixel value in $R$ and $z_{m}$ is the median value in this neighborhood. Rotational Invariance Uniform (RIU) is defined as,

$$
M G B P_{P, R}^{r i u 2}=\left\{\begin{array}{cl}
\sum_{p=0}^{P-1} \delta\left(z_{p}-z_{m}\right) & \text { if } U\left(M G B P_{P, R}^{U}\right) \leq 2 \\
P+1 & \text { otherwise }
\end{array}\right.
$$

where

$$
\left(M G B P_{P, R}^{U}\right)=\left|\delta\left(z_{p-1}-z_{m}\right)-\delta\left(z_{0}-z_{m}\right)\right|+\sum_{p=0}^{P-1}\left|\delta\left(z_{p}-z_{m}\right)-\delta\left(z_{p-1}-z_{m}\right)\right|
$$

After obtaining the local patterns in the processed image, we compute the MGBP hisotgram at each pixel within $N \times N$ centered patch. These histograms provide MGBP descriptors. 




Fig. 4. Evaluation scheme.

\subsection{Nerve classification}

In the classification process, each pixel of the segmented foreground is labeled as belonging, or not, to a nerve tissues. For that purpose we use the SVM algorithm to identify the nerve pixels. SVM classifier [18] is based on intuitive geometric principles, aiming to define an optimal hyperplane in the training data so that minimum expected risk is achieved. SVM is widely used for image segmentation and classification showing very powerful discriminative properties. In this study, the linear kernel $\left.K\left(x, x^{\prime}\right)=\left(<x, x^{\prime}\right\rangle\right)^{n}$ has shown the best performances compared to the non linear ones.

\section{Experimentation}

The experiments were conducted over 173 ultrasound images of the median nerve extracted from three US videos of patients. Data set was obtained in real conditions at Médipôle Garonne clinic (Toulouse, France). The ground truth was marked by regional anesthesia experts.

In the first stage the k-means segmentation was performed over all the images to segment the foreground (see section 2.1), then the classification process was applied over the foreground to extract the target region. For that purpose, the dataset were separated into three groups, each one containing a set of the US images of one patient; the images size is $633 \times 418$. We use one group for learning the model and the remaining ones for the tests. The cross validation procedure was used to generate the learning and testing sets (see Fig. 4). For each couple of learning/testing set, the SVM algorithm has been applied to classify the pixels. Specificity, sensitivity and accuracy were used to measure the performance of the tested methods,

$$
\text { Accuracy }: \text { Acc }=\frac{S n+S p}{2}, \text { Sensitivity }: S n=\frac{t p}{t p+f n}, \text { Specificity }: S p=\frac{t n}{t n+f p}
$$

where $t p, t n, f p, f n$ are respectively true positives, true negatives, false positives and false negatives. In order to illustrate the effectiveness of the proposed approach for the nerve detection, we have conducted comparative experiments with most of the popular methods of texture analysis in ultrasound images, such as the Gabor descriptor, LBP, MBP, Wavelet, GLCM and LGBP with different parameters. The Gabor filters have been generated using different combination of parameters for the orientation $\theta=(0, \pi / 4, \pi / 2,3 \pi / 4)$ and the scale $s=(1,2,3)$. The experiments of LBP and MBP have been generated using a several values for radius $R$ and neighborhood $P$ (i.e. $R=(1,2,3)$ and $P=(8,16,24)$ ). Different Wavelet families have been applied to our data, in this experiment we kept 
the best parameters of each family. The GLCM descriptor was also tested with different orientations, whereas the distance of one pixel showed better results. Finally, it is also important to study the influence of the patch size, for our experiments the size $35 \times 35$ produced the best classification scores.

\subsection{Evaluation of the texture methods}

In this section we measure the performance of several state-of-the-art methods, by varying the parameters of each method as shown in Tables 1, 2, 3, 4. From these tables, it can be seen that the best accuracy score for each method (bold type in the table) depends on the specific set of parameters. For the Gabor descriptor, scale $=1$, orientation $(\theta=\pi / 2)$ and frequency $(\lambda=1)$ yielded the highest value $(70.89 \%)$. For the LBP and MBP, the best parameters are $L B P_{\text {riu }}^{3,24}$ and $M B P_{\text {riu }}^{8,16,24}$, respectively. For the Wavelet descriptor, the symlets wavelet, where the number of vanishing moments is five (sym5) [2] and level 3 produced $63.92 \%$. GLCM yielded an accuracy of $62.45 \%$ for distance $=1$ and orientation $=0$. The best classification accuracy scores were obtained with Gabor and MBP descriptors produced the better accuracy (70.89\% and $70.50 \%$ ) compared to the traditional techniques. Best performance were obtained when these two methods were combined. Table 4 shows the MGBP and LGBP classification accuracies. It can be observed that MGBP achieves higher average accuracy $(71.34 \%)$ than LGBP. The largest difference is about $8.79 \%$ when $R=1$ and $P=8$.

\begin{tabular}{|c|c|c|c|c|c|c|c|c|c|c|c|}
\hline Params & \multicolumn{3}{|c|}{ Scale $=1$} & Params & \multicolumn{3}{|c|}{ Scale $=2$} & Para & \multicolumn{3}{|c|}{ Scale $=3$} \\
\hline$\theta$ & Acc & Sn & Sp & $\theta$ & Acc & Sn & Sp & $\theta$ & Acc & Sn & Sp \\
\hline 0 & 66.13 & 52.36 & 79.9 & 0 & 65.37 & 50.58 & 80 & 0 & 63.91 & 46.96 & 80.86 \\
\hline$\pi / 4$ & 64.88 & 49.30 & 80.4 & $\pi / 4$ & 67.64 & 52.68 & 82.5 & $\pi / 4$ & 70.17 & 8.03 & 82.31 \\
\hline$\pi / 2$ & 70.89 & 58.47 & 83.32 & $\pi / 2$ & 68.04 & 53.97 & 82.1 & $\pi / 2$ & 66.22 & 45.32 & 87.12 \\
\hline $3 \pi / 4$ & 70.48 & 51.19 & 89.77 & $3 \pi / 4$ & 68.91 & 49.70 & 88.12 & $3 \pi / 4$ & 60.36 & 38.35 & 82.37 \\
\hline
\end{tabular}

Table 1: Classification results for different parameters of Gabor

\begin{tabular}{|c|c|c|c|c|c|c|c|c|}
\hline \multicolumn{3}{|c|}{ Params } & \multicolumn{3}{c|}{ LBP } & \multicolumn{3}{c|}{ MBP } \\
\hline Type & $\mathrm{R}$ & $\mathrm{P}$ & Acc & Sn & Sp & Acc & Sn & Sp \\
\hline u2 & 1 & 8 & 65.24 & 86.90 & 43.59 & 37.78 & 23.44 & 52.12 \\
ri & 1 & 8 & 66.14 & 87.43 & 44.85 & 45.72 & 35.03 & 56.40 \\
ri & 2 & 16 & 66.15 & 87.43 & 44.86 & 46.65 & 24.12 & 69.19 \\
riu 2 & 1 & 8 & 66.14 & 87.43 & 44.85 & 58.77 & 47.11 & 70.42 \\
riu 2 & 2 & 16 & 66.12 & 87.41 & 44.83 & 56.65 & 33.33 & 79.98 \\
riu 2 & 3 & 24 & $\mathbf{6 4 . 9 1}$ & 86.39 & 43.42 & 53.59 & 32.61 & 74.57 \\
riu 2 & $1,2,3$ & $8,16,24$ & 64.42 & 52.21 & 76.63 & $\mathbf{7 0 . 5 0}$ & 65.87 & 75.14 \\
\hline
\end{tabular}

Table 2: Classification results for different parameters of LBP and MBP 


\begin{tabular}{|c|c|c|c|c|c|c|c|}
\hline Wavelet & \multicolumn{3}{|c|}{ Level $=3$} & GLCM & \multicolumn{3}{|c|}{ Dist $=1$} \\
\hline families & Acc & Sn & Sp & Orientation & Acc & Sn & Sp \\
\hline & 63.74 & 60.29 & 67.1 & 0 & 62.45 & 79.04 & \\
\hline & $6:$ & 60 & & $\pi / 4$ & & 79.61 & 9.0 \\
\hline & & & & $\pi / 2$ & & 9.46 & 1.2 \\
\hline coif 1 & 63.92 & 61.15 & 66.33 & $3 \pi / 4$ & 60.06 & 79.58 & 0.5 \\
\hline
\end{tabular}

Table 3: Classification results for different Wavelet families and GLCM

\begin{tabular}{|c|c|c|c|c|c|c|c|}
\hline \multicolumn{2}{|l|}{ Params(riu2) } & \multicolumn{3}{|c|}{ LGBP } & \multicolumn{3}{c|}{ MGBP } \\
\hline R & P & Acc & Sn & Sp & Acc & Sn & Sp \\
\hline 1 & 8 & 61,99 & 70.97 & 53.02 & $\mathbf{7 1 . 3 4}$ & 82.38 & 60.30 \\
2 & 16 & $\mathbf{6 2 . 5 5}$ & 44.42 & 80.68 & 57.01 & 50.29 & 63.73 \\
3 & 24 & 60.54 & 35.54 & 89.54 & 61,15 & 34.61 & 87.69 \\
$1 \_2$ & $8 \_16$ & 61.14 & 68.97 & 53.31 & 68,20 & 77.39 & 59.01 \\
$1 \_3$ & $8 \_24$ & 61.82 & 66.63 & 57.01 & 63,72 & 74.01 & 53.43 \\
$2 \_3$ & $16 \_24$ & 60.12 & 46.63 & 74.17 & 60,42 & 40.39 & 80.45 \\
1_2_3 & 8_16_24 & 66.99 & 70.97 & 53.02 & 66.48 & 70.05 & 62.45 \\
\hline
\end{tabular}

Table 4: Classification results for different parameters of LGBP and MGBP

\subsection{Overall comparison}

In this part, we compare the proposed method (MGBP) with the other techniques previously described, using only the best scores of each technique. Fig. 5 shows qualitative and quantitative experimental results. From this figure, we can see that MGBP reachs the highest correct classification rates (71.34\%) among all the other approaches. The qualitative results demonstrate that MGBP provides better accuracy for nerve detection. In general, the combination of MBP and Gabor filter is more accurate than the original MBP or Gabor as a single feature. MGBP attains the best results and realizes a good tradeoff between the Sensitivity and Specificity, which enable an accurate nerve detection. However, the experimental results shows that the texture of nerve as hypoechoic tissues is hard to detect using mono model feature.

This demonstrates that MGBP is stable and robust for US images and proved to be a good descriptor for US images.

\section{Conclusion}

In this paper we have proposed a new technique for nerve detection in ultrasound images using two phases: the k-means clustering for foreground segmentation and the texture analysis to extract the nerve region amid the foreground hyperechoic tissues. The nerve identification presents the most critical part of this work. As the nerve presents some interesting textural properties, we focused on the texture analysis. A new method (MGBP) based on MBP and Gabor filter have been proposed as texture descriptor. The experimental evaluation on real data, showed that MGBP enables efficient detection of the nerve region compared to the tested state-of-the-art methods. This is due to the fact that MBP and Gabor 




Fig. 5. Comparison of classification results of the proposed method and the best method of analysis texture.

enhance the texture profile in the noisy data, which increase the robustness of MGBP descriptor with respect to the speckle noise and other artifacts in the US images. In the future, we will explore the possibilities to extend the detection to others types of nerves.

\section{References}

1. D. Boukerroui, O. Basset, A. Baskurt, and G. Gimenez. A multiparametric and multiresolution segmentation algorithm of 3-d ultrasonic data(hrlk). IEEE transactions on ultrasonics, ferroelectrics, and frequency control, 48(1):64-67, 2001.

2. I. Daubechies. Ten lectures on wavelets. SIAM, pages 258-259, 1992.

3. J.G. Daugman. Uncertainty relation for resolution in space, spatial frequency, and orientation optimized by two-dimensional visual cortical filters. Journal of the Optical Society of America, 2:1160-1169, 1985.

4. S. Ghose, A. Oliver, R. Mart, X. Llad, J. Freixenet, J. Villanova, and F. Meriaudeau. Prostate segmentation with local binary patterns guided active appearance models. Medical Imaging: Image Processing, 2011.

5. A. Hafiane, G. Seetharaman, K. Palaniappan, and B. Zavidovique. Rotationally invariant hashing of median binary patterns for texture classification. International conference on image analysis and recognition, pages 619-629, 2008.

6. R. M. Haralick, K. Shanmugam, and I. Dinstein. Textural features for image classification,. IEEE Trans. Syst. Man Cybern, 3:610-621, 1973.

7. A. Khanna, M. Sood, and S. Devi. Us image segmentation based on expectation maximization and gabor filter. International Journal of Modeling and Optimization, 2, 2012.

8. C. Knoll, M. Alcaniz, C. Monserrat, V. Grau, and M. C. Juan. Outlining of the prostate using snakes with shape restrictions based on the wavelet transform (doctoral thesis: Dissertation). Pattern Recognition, 32:1767-1781, 1999.

9. G. Malathi and V. Shanthi. Histogram based classification of ultrasound images of placenta,(hrlk). International Journal of Computer Applications, 2001.

10. P. Marhofer, W. Vincent, and S. Chan. Ultrasound-guided regional anesthesia: Current concepts and future trends. Journal of Clinical Anesthesia, 105, 2007.

11. A. Noble. Ultrasound image segmentation: A survey,. IEEE Transactions on Medical Imaging, 25:28, 2006. 
12. T. Ojala, M. Pietikinen, and T. Menp. Multiresolution grayscale and rotation invariant texture classification with local binary patterns. IEEE Trans. Pattern Analysis and Ma-chine Intelligence, 24:971-987, 2000.

13. D. Shen, Y. Zhan, and C. Davatzikos. Segmentation of prostate boundaries from ultrasound images using statistical shape model,. IEEE Transactions on Pattern Analysis and Machine Intelligence, 2003.

14. J. Stoitsis, S. Golemati, N. Tsiaparas, and K. S. Nikita. Texture characterization of carotid atherosclerotic plaque from b-mode ultrasound using gabor filters. Proc IEEE Conf. Eng. Med. Biol. Soc., pages 455-458, 2009.

15. L. Tesar, D. Smutek, and J. iskra. Genetic algorithms for thyroid gland ultrasound image feature reduction(haralick). Lecture Notes in Computer Science Volume, 3612:841-844, 2005 .

16. E. Thouin, A. Hafiane, P. Vieyres, N. Xylourgos, G. Triantafyllidis, and G. Papadourakis. Nerve region segmentation for ultrasound guided local regional anaesthesia (lra). Mediterranean Conference on Information Systems, 2011.

17. Nikolaos N. Tsiaparas, Spyretta Golemati, Ioannis Andreadis, and John S. Stoitsis. Comparison of multiresolution features for texture classification of carotid atherosclerosis from b-mode ultrasound. Transactions on information technology in biomedicine, 15:1, 2011.

18. V. Vapnik. Statistical learning theory. Wiley-Interscience, 1998

19. Glenn E. Woodworth, Elliza M. Chen, Jean-Louis E. Horn, and Michael F. Aziz. Efficacy of computer-based video and simulation in ultrasound-guided regional anesthesia training. Journal of Clinical Anesthesia, 2014.

20. A. Zaim, Y. Taeil, and R. Keck. Feature based classification of prostate us images using multiwavelet and kernel svm. In Proceedings of International Joint Conference on Neural Networks, pages 278-28, 2007.

21. W. Zhang, S. Shan, W. Gao, X. Chen, and H. Zhang. Local gabor binary pattern histogram sequence (lgbphs): A novel non-statistical model for face representation and recognition. IEEE Int',l Conf. Computer Vision, pages 786-791, 2005. 